\title{
Dose optimization of ceftriaxone-vancomycin combination using fractional inhibitory concentration kinetics in resistant bacteria
}

\author{
Vishnu D Sharma*, Aman Singla, Manu Chaudhary, Mukesh Kumar, Anuj Bhatnagar, \\ Shailesh Kumar, Manish Taneja* \\ Venus Medicine Research Centre, Hill Top Estate, Bhatoli Kalan, H.P., India \\ *Corresponding Authors. E-mail: vishnudutt070788@gmail.com \& manishtaneja24@gmail.com; Tel.: +91-9871681044 \\ Received: May 02, 2016; Revised: July 12, 2016; Published: September 30, 2016
}

\begin{abstract}
The notorious Staphylococcus aureus resistant strains with ever changing resistance patterns have limited treatment options and have led to substantial number of deaths. Almost dried antibiotic pipeline has led us to look into combinations of already approved antibiotics for tackling rising incidence of antibacterial resistance. Recommended use of vancomycin and ceftriaxone together for treating severe infections involving resistant $S$. aureus is limited by dose adjustments and different dose frequencies. We have developed a pharmacodynamically synergistic fixed dose combination (FDC) of ceftriaxone and vancomycin (2:1), for eliminating individual component dose adjustments and frequencies. For identification of optimum exposureresponse of FDC, one compartment in vitro system was used for dose escalation, fractionation and doseresponse studies. The in-silico pharmacokinetic/pharmacodynamic (PK/PD) modeling, simulations and validations were done. The results suggested \% $T>M I_{\text {comb }}$ (percentage of time fractional inhibitory concentrations of the drugs combined remained above the $\mathrm{MIC}_{\text {comb }}$ [minimum inhibitory concentration for FDC]) followed by $A U C_{\text {comb }} / M I C_{\text {comb }}$ (ratio of area under fractional inhibitory curves to $M I C C_{\text {omb }}$ ) can predict the exposure (dose of $F D C$ )-response (reduction in bacterial load) relationships effectively ( $r 2>0.9$ ). Total exposure of $6 \mathrm{~g}$ in two divided doses (3 $\mathrm{g}$ each) was identified to be optimum. Monte Carlo simulations were performed to evaluate the effect of increasing doses against different MICs. Clinical breakpoint of the FDC was identified to be $4 \mu \mathrm{g} / \mathrm{mL}$, which was 2 fold higher than that of vancomycin suggesting better antibacterial coverage.
\end{abstract}

\section{Keywords}

PK/PD modelling; Fixed dose combination; Monte Carlo simulations; Antibacterial resistance

\section{Introduction}

The bacteria methicillin-resistant Staphylococcus aureus (MRSA) is one of most common pathogen responsible for nosocomial infections. It is associated with an increased risk of mortality and morbidity, requiring prolonged duration of treatment and increased cost [1]. It is reported that out of 10,800 deaths per year by infections in United States, 5,500 deaths are linked to MRSA. The prevalence of MRSA is extrapolated to 1 billion people by year 2022 [1].

Vancomycin is the empirical choice for treating these infections. However, extensive vancomycin use has led to emergence of MRSA with reduced vancomycin susceptibility. In 2006, the minimum inhibitory 
concentration (MIC) breakpoints for S. aureus were lowered (from $\leq 4 \mu \mathrm{g} / \mathrm{mL}$ to $\leq 2 \mu \mathrm{g} / \mathrm{mL}$ for "susceptible," from 8-16 $\mu \mathrm{g} / \mathrm{mL}$ to $4-8 \mu \mathrm{g} / \mathrm{mL}$ for "intermediate," and from $\geq 32 \mu \mathrm{g} / \mathrm{mL}$ to $\geq 16 \mu \mathrm{g} / \mathrm{mL}$ for "resistant") and vancomycin was less likely to respond when MICs were $\geq 4 \mu \mathrm{g} / \mathrm{mL}$ [2]. The clinical relevance of change in breakpoint was further highlighted by the observation wherein subjects with high grade infections required high vancomycin MICs leading to therapy failures or increase in mortality. Mechanistically, vancomycin binds to the terminal $\mathrm{D}$-alanyl-D-alanine moieties of the $\mathrm{N}$-acetyl muramic acid/N-acetyl glucosaminepeptides, subsequently prevents the action of penicillin binding proteins and stops peptidoglycan growth (leading to bacterial killing). However, in case of resistant bacteria (S. aureus), the last D-alanyl moiety is replaced by D-lactate, which makes bacteria less permeable to vancomycin (vancomycin entrapment and increase membrane thickness). This "permeability-mediated" resistance manifested clinically by treatment failure or delayed resolution of $S$. aureus infections [3,4]. Addition of an antibiotic that can resolve the permeability issue is a rationale choice. It was demonstrated by our group previously [5] that ceftriaxone plus vancomycin formulation ( $2: 1 \mathrm{w} / \mathrm{w}$ ratio), exploits the virgin territory of the vancomycin resistant bacteria i.e. penicillin binding proteins. Ceftriaxone bind irreversibly with these proteins and thus inhibit the transpeptidation step of peptidoglycan synthesis to stop bacterial cell wall growth. Ceftriaxone-induced fragility in the peptidoglycan layer increases the penetrability of the vancomycin and restores (to some extent) its affinity towards the exposed D-alanine moieties. The 'double insult' caused by the antibiotic FDC makes bacterial cell susceptible to death.

After pharmacodynamic selection of drugs combination to combat MRSA, the second step is to optimize dose regimens using their pharmacokinetic/pharmacodynamic (PK/PD) relationships [6,7]. The dosing regimens of antibiotics are based on MIC estimates (a surrogate PD marker for antibacterial response characterization) and the quantification of exposure (changes in concentration of individual components of dose combination) - response (the reduction of bacterial count) relationship [6,7]. The exposure-response relationships help in evaluating the PK/PD indices [8,9] and further identifying the efficacy drivers for understanding the potential activity of antibacterial agents. The three PK/PD indices that are routinely used for explaining the therapeutic efficacy in terms of achieving MIC and mechanism based action of antibiotics include: 1) \% T>MIC time (\% time for which the levels of antibiotic in serum/plasma exceed the MIC); 2) Area under the concentration-time curve (AUC)/MIC ratio; 3 ) Peak plasma concentration $\left(C_{\max }\right) / \mathrm{MIC}$ ratio [10-12]. Maximum literature for these kinds of PK/PD studies is available for single antibacterial agents $[13,14]$; the effect of combination therapy on the PK and PD, and subsequently on PK/PD indices is very limited [15].

The active components of the fixed dose combination (FDC) i.e. vancomycin (AUC/MIC ratio is the preferred index) and ceftriaxone (\%T>MIC is the preferred index) have similar PK profiles but their optimized PK/PD indices correlative of therapeutic efficacy are different. The parameters which are usually used to show antibiotic interactions for combination therapy are FIC (fractional inhibitory concentration) indices. Through this approach, the synergism or antagonism expressed as the maximum effect of the antibiotic combination can be calculated (FIC index identified for this combination was $<0.5$ suggesting synergistic pharmacodynamic action [5], and estimations of PK/PD index on the basis of FICs are considered as composite predictors.

The purpose of the present study is to use in-silico PK/PD modeling and simulations strategies to identify the FIC based PK/PD indices that can predict the therapeutic efficacy of combination therapy and further help in optimization of dosage regimen (Figure 1) [16]. After identification of best PK/PD index driving the therapeutic outcome, Monte Carlo simulations were performed to understand the clinical 
benefit of the FDC and 1000 subjects' simulated data was generated using population PK parameters of individual components of the FDC [17]. The PTA (probability of target attainment) of PK/PD index in the simulated data was calculated and the clinical breakpoint of the FDC was identified. The breakpoint analysis will not only help us in optimizing the use of antibiotic combination, but also in identifying the differences in resistance pattern for ceftriaxone/vancomycin (2/1) in comparison to vancomycin alone.

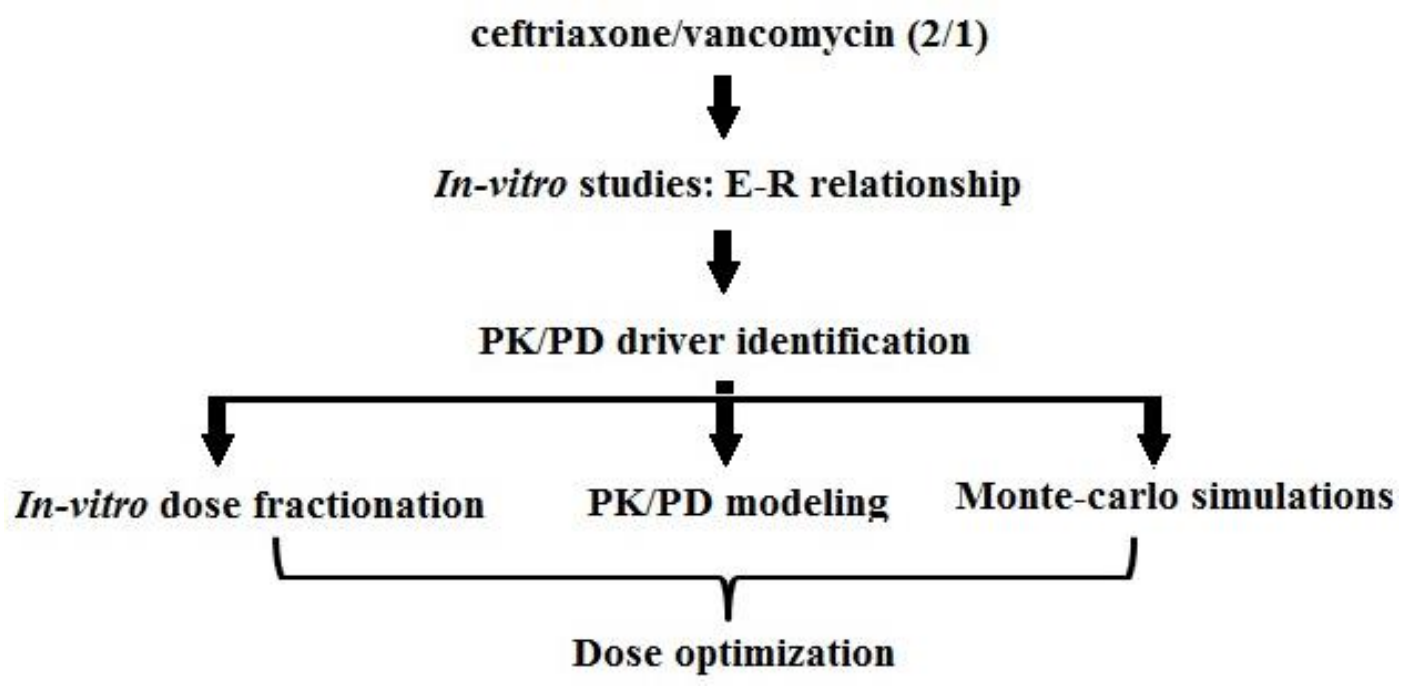

Figure 1. Schematics of the approach used in the study. In vitro studies were performed to study exposureresponse relationships and PK/PD driver identification. Using the information of in vitro studies, three approaches i.e. in vitro studies, PK/PD modeling and Monte Carlo simulations were employed for dose recommendations.

\section{Materials and methods}

Antibiotics, bacteria, and media

The FDC of ceftriaxone/vancomycin (2/1) vials (Vancoplus) were obtained from Venus Remedies Ltd, H.P., India. Genotypically characterized MRSA clinical isolates with reduced susceptibility to Vancomycin were taken from clinical isolate bank of Venus Medicine Research Centre, Baddi. The media Mueller-Hinton broth (Becton Dickinson, Sparks, MD) was used to perform all in vitro studies involving MIC determination, bacterial kinetics, dose ranging, fractionation, and response studies.

\section{Quantification of bacteria and antibiotic}

The bacterial density (CFU [colony forming units]) and MIC determinations were conducted according to Clinical Laboratory Standard Institute guidelines [18]. The FIC index was determined using checkerboard method [5]. The media samples were analyzed for ceftriaxone and vancomycin concentrations using high performance liquid chromatography, as mentioned in our previous article [19].

\section{One-compartment chemostat infection model}

Single-compartment chemostat infection system as described previously $[14,20,21]$ was used for the in vitro modeling of the FDC. In summary, the chemostat system assembly consisted of a $500 \mathrm{~mL}$ glass central reservoir chamber with five ports for the addition and removal of media via silicone tubes connected to peristaltic pumps, injection of drug (antibiotic combination) solution, and removal of medium samples. Prior to each experiment, MRSA colonies were grown overnight to obtain a starting inoculum of 108 $\mathrm{CFU} / \mathrm{mL}$ in $500 \mathrm{~mL}$ central reservoir flask containing media. An aqueous solution of FDC was prepared. The 
"in" and "out" flow rates from the central reservoir were adjusted to obtain half-life of $\sim 5-6 \mathrm{~h}$ [22-24]. Samples from the central reservoir were collected at different time points from 0 to $24 \mathrm{~h}$ post-FDC addition. The samples were analysed for individual component concentrations and CFU determination for bacterial densities. The bacteria growth control experiments were also performed using the same experimental set-up without adding any drug. Net PD effects of all doses of FDC were then reported after accounting the bacterial dynamics of the growth control experiment. Pre and post FDC exposure, MICs were determined for evaluating any changes in MICs due to FDC exposure. Also the PK parameters calculated in in vitro chemostat infection model for individual drugs were in concordance with in vivo literature values (Table 1) supporting the validity of in vitro systems.

Dose escalation, fractionation and response curve studies using in vitro chemostat model

The drug concentrations were varied from 0.5-200 folds MICs to determine the most efficacious exposure based on their PK/PD parameters. The best exposure from dose escalation study was then fractionated twice-a-day and thrice-a-day to determine a dosage regimen of antibiotic which can enhance bacterial killing at lower concentration. The best dosage regimen was then used to construct dose response curve. The dosage regimen at $75,50,38$, and $25 \%$ of original concentration were utilized and the corresponding change in bacterial density in $24 \mathrm{~h}$ was measured. The dose response curve was plotted between the dose percentage and log reduction in bacterial density and EC50 was calculated.

\section{Pharmacokinetic analysis}

One compartment model was used to fit the time concentration values and PK parameters of ceftriaxone and vancomycin were calculated. The parameters included the area under the plasma concentration-time curve (AUC), maximal concentration reached (the peak; $C_{\max }$ ), elimination rate constant $\left(K_{\mathrm{e}}\right)$, half-life $\left(t_{1 / 2}\right)$, volume of distribution $(\mathrm{VD})$, and clearance $(\mathrm{CL})$. These parameters were calculated for all dose escalations and dose fractionation studies. The parameters obtained from concentration profile data were compared with reported population PK parameters in the literature (Table 1).

\section{Pharmacokinetic/pharmacodynamic analysis}

The FIC curves for ceftriaxone and vancomycin combinations were generated. Briefly, the concentrations of each antibiotic at every time-point were divided by their respective MIC contributions towards $\mathrm{MIC}_{\text {comb }}$ to obtain $\mathrm{FIC}_{\text {ceftriaxone }}$ and $\mathrm{FIC}_{\text {Vancomycin }}$ values. These values were added $\left(\mathrm{FIC}_{\text {comb }}\right)$ for each time points and plotted against time. The resultant FIC-time profile was fitted to one compartment model to obtain PK/PD parameters i.e. $\mathrm{AUC}_{\text {comb }}, \mathrm{C}_{\text {max-comb }} \% \mathrm{~T}>\mathrm{MIC}_{\text {comb }}$ and $\% \mathrm{~T}>\mathrm{FIC} \mathrm{C}_{\text {comb }}[9,16]$. In addition, The $\mathrm{PK} / \mathrm{PD}$ indices i.e. AUC/MIC, $C_{\max } / \mathrm{MIC}$ and $\% \mathrm{~T}>\mathrm{MIC}$ were also calculated for both ceftriaxone and vancomycin using their respective PK parameters. 


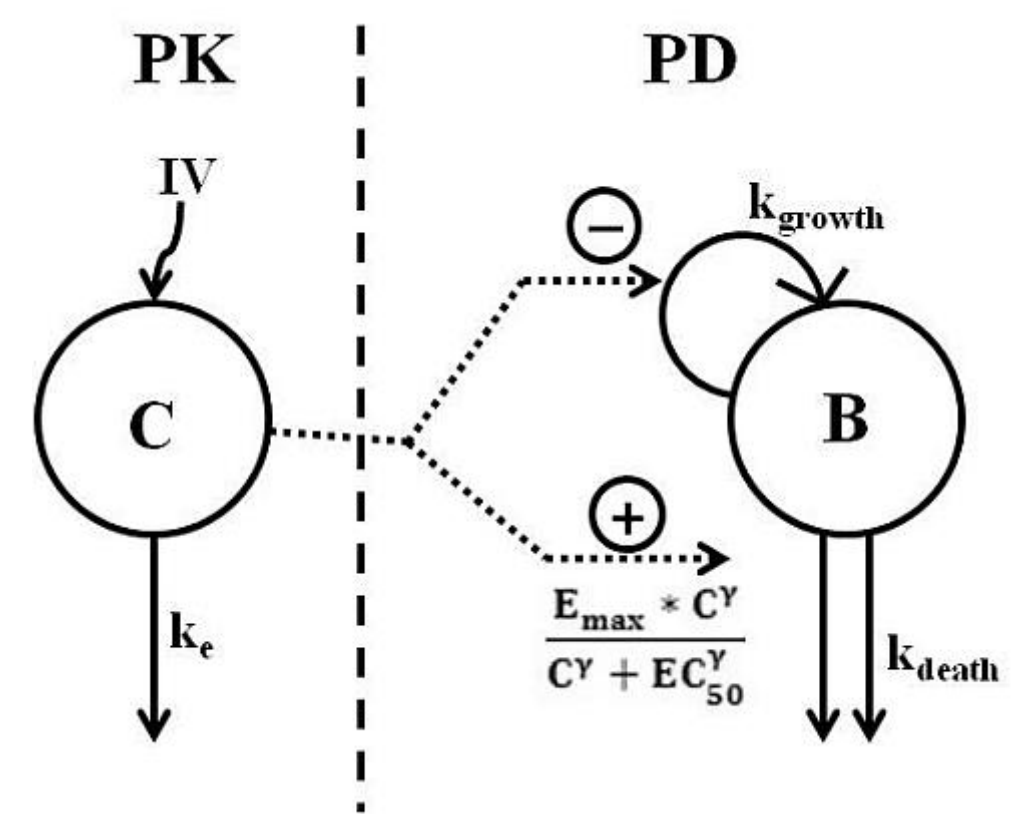

Figure 2. The inter-relationship between the time dependent changes in drug concentrations (PK-intravenous (IV) infusion) and the bacterial growth inhibition/bacterial killing (PD) after antibacterial treatment. The parameters are $C$, the concentration of combination or individual component of FDC in central drug compartment; $k_{\mathrm{e}}$, drug elimination rate constant; $k_{\text {growth }}$ and $k_{\text {death }}$, rate constants for multiplication and degradation of bacteria, respectively and $B$ is bacterial load at a given time point.

\section{Semi-mechanistic pharmacokinetic/pharmacodynamic modeling and validation}

Bacterial modeling (control experiments): The bacterial kinetics determination involves modeling of a single bacterial compartment (B) with first-order rate constants for bacterial multiplication $\left(k_{\text {growth }}\right)$ and bacterial death $\left(k_{\text {death }}\right)$ [25]. The equation 1 explains the observed exponential growth of bacteria until it reaches a stationary bacterial level without the addition of antibiotics (control experiments) and where $B$ and $B_{\max }$ are initial bacterial density and maximum bacterial density [26].

$\frac{d B}{d T}=\left(K_{\text {net }}\right) *\left(1-\frac{B}{B_{\max }}\right) * B$

Pharmacokinetic/pharmacodynamic modeling: The bacterial load was evaluated at 8, 9.5, 12 and $24 \mathrm{~h}$ post antibiotic exposures. The 8,12 and 24 time-points were selected to evaluate bacterial load in case of thrice-a-day, twice-a-day and once-a-day dose regimens respectively; whereas, $9.5 \mathrm{~h}$ time-point was selected for internal evaluation as the second inoculum was added to in vitro system at $9.5 \mathrm{~h}$ to mimic harsher in vivo conditions. The relationship between the effect and the corresponding PK/PD indices was evaluated according to a sigmoidal $E_{\max }$ type function as described in equation $2[9,6]$. The $E_{\max }$ model describes the concentration-effect relationship in terms of a baseline effect or $E_{0}$, hence taking into account the baseline condition which can significantly affect the maximum activity of antibiotic i.e. $P D_{\max }$ a descriptor of direct response of antibiotic. Additionally, the function asymptotes to an upper limit of inhibition by an antibiotic on bacterial load.

$E=E_{0}-\frac{P D_{\max } * X^{\text {nulu }}}{X^{\text {hill }}+E X_{50}^{\text {hill }}}$

where, $E$ is the PD endpoint i.e. bacterial density calculated as change in $\log _{10} \mathrm{CFU} / \mathrm{mL}$ after $8,9.5,12$ or 24 $\mathrm{h}$ of treatment, $E_{0}$ is the baseline effect i.e. PD endpoint without antibiotic treatment; $X$ is PK/PD index; 
$\mathrm{PD}_{\max }$ is a maximum effect; $E \mathrm{X}_{50}$ is magnitude of $X$ that is needed to achieve $50 \%$ of the $\mathrm{PD}_{\max }$; Hill is the sigmoidicity factor, reflecting the steepness of the relationship.

All data from the different dosing regimens was fitted to above-mentioned model (equation 2). Curve fitting was performed in GraphPad Prism (version 4.01, GraphPad software, San Diego, CA) using the nonlinear regression analysis. The coefficients of determination $\left(R^{2}\right)$, sigmoidicity factor, and the visual inspection of observed versus predicted values graphs were used to select the best PK/PD index and the best predicted endpoint of antibacterial effect.

Anti-bacterial-PK/PD modeling: Basic assumption for the dependence of antibacterial effect is generally based on non-linear relationship with concentration data of antibiotics. Higher the sigmoidicity factor, lesser is the predictability of PD effect with respect to PK/PD index. The concentration-effect relationships were incorporated in the bacterial model (equation 1) to predict the bacterial count from PK/PD model [26-29] (equation 3). In the constructed model, the antibacterial effect of FDC of ceftriaxone/vancomycin is hypothesized as a combination of bacterial growth inhibition $\left(k_{\text {growth }}\right)$ and bacterial killing enhancement $\left(k_{\text {death }}\right)$ (Figure 2).

$$
\frac{d B}{d T}=K_{n e t} * B-\frac{E_{\max } * C^{Y}}{C Y+E C_{50}^{Y}} * B
$$

\section{Model evaluation}

All data from the different drug exposure and dosage regimen was fitted to above-mentioned model (equation 3 ) for the time period of 8 and $24 \mathrm{~h}$ respectively using SCIENTIST (MicroMath, version 3.0, Saint Louis, Missouri, USA). The $95 \%$ confidence intervals were drawn for all the predicted values and compared with the observed values (obtained from in vitro system). Additionally, the coefficients of determination (R2) between predicted and observed data values were determined and evaluated for the validation of the antibacterial-PK/PD model.

\section{Monte Carlo simulations}

Monte Carlo simulations for 1000 adult subjects were performed to determine how likely the FDC dose of $0.75,1.5,3,6$, and 9 gm would achieve $\mathrm{AUC}_{\text {comb }}>400$ and $60 \% \mathrm{~T}>\mathrm{MIC}_{\text {comb }}$ at different values of MIC i.e. $1,4,8,16,3,64 \mu \mathrm{g} / \mathrm{mL}$. The population PK parameter utilized in simulations was clearance of $0.88+/$ $0.431 \mathrm{~L} / \mathrm{h}$ [30] and $4.8+/-1.9 \mathrm{~L} / \mathrm{h}$ [31] for ceftriaxone and vancomycin, respectively. The patient weight is an important covariate on drug's clearance, which was assumed to be $70 \mathrm{~kg}$ in the analysis. For $\mathrm{AUC}_{\text {comb, }}$ each dose was divided by the clearance values of simulated subjects at all MIC values. The PTA for AUC comb was defined as percentage of the simulated subjects in which $A U C_{\text {comb }}$ was more than desired values (i.e. 400 in present studies). For $\% \mathrm{~T}>\mathrm{MIC}_{\text {comb }}$, the concentration-profiles were generated using $\mathrm{CL}$ and $V_{\mathrm{d}}$ of each simulated subjects and \% $>\mathrm{MIC}_{\text {comb }}$ was calculated for all simulated subjects for different doses at all MIC values. The PTA was then defined as percentage of simulated subjects showing $\% \mathrm{~T}>\mathrm{MIC}_{\text {comb }}$ of more than 60.

\section{Data analysis}

Descriptive statistics were used for reporting all PK variables and summary tables were prepared using mean, standard deviation (SD), median, and range (whichever appropriate). Log transformed data was used wherever applicable. The statistical analysis was done using GraphPad Prism (version 4.01, GraphPad software, San Diego, CA). Non-linear regression analysis and logistic regression was done for bacterial 
kinetics using SCIENTIST (Micromath, version 3.0, Saint Louis, Missouri, USA). Monte Carlo simulations were done using macros in MS excel 2010.

\section{Results and Discussion}

The major problem with antibacterial resistance is limited treatment options. With limited antibiotic research, FDC of already approved antibiotic may still provide certain answers to tackle the resistance [32]. However, the major concern with antibiotic FDCs utilization is their dose and frequency of dosing. Focusing on dose optimization, it mainly depends upon the pharmacokinetic, and pharmacodynamic properties of a drug. The complexity increases with addition of another active drug in the formulation, similar to the one presented in the study. The current formulation is a FDC of ceftriaxone and vancomycin in 2:1 (w/w), results in reduction of combined MIC from 16 to $4 \mu \mathrm{g} / \mathrm{mL}$ against a MRSA bacterial strain used for this study. The FIC index was $<0.5$ again confirms the synergism offered by FDC [5]. The present study was focused on dose optimization of the FDC using in vitro studies, PK/PD modeling and Monte Carlo simulations.

\section{Exposure-response relationship assessment using in vitro studies}

The in vitro models allow good prediction of in vivo bacterial growth in vivo, helps in comparing different dosing regimens of drugs, and thus play an important role in dose optimization [21]. The selection of a specific in vitro model is determined by the objectives of a PK/PD study. The usual method for testing in vitro antibiotic combinations involves the exposure of a given bacterial inoculum to a static concentration of the anitbiotics. However, bacteria at the site of an infection are exposed in vivo to varying concentrations of antibiotics. Therefore, in the present study, we have employed dynamic dilution in vitro system i.e. one-compartment chemostat infection model to mimic the in vivo conditions with respect to the changing drug and medium much more closely. Chemostat infection model provides optimal bacterial growth conditions by replenishing nutrients via fresh media, and removing drug/wastes/old media, hence mimicking closely the biological dynamics of bacteria during human infection. The in vitro system simulates 1st order drug elimination leading to exponential decrease in drug concentration. As both vancomycin and ceftriaxone concentration-time plots follow one compartment PK model, the model was apt to fit the first order kinetics of both drugs.

The in vitro model for dose optimization requires PK and PD inputs to evaluate the PK/PD of a given exposure of drug against an infection. For PK input, the PK parameters reported in literature for ceftriaxone and vancomycin were employed (Table 1). Focusing on PD target, MRSA isolates were selected as a prototype of MRSA infections. Microbiological studies were then performed for the selection of the target strain against which exposure-response relationship can be evaluated. It was observed that MRSA strain of MIC $4 \mu \mathrm{g} / \mathrm{mL}$ exhibits dose-dependent behaviour against the FDC, which underlines its suitability in exposure-response studies (results not shown). The PD effect was defined as logarithmic reduction in MRSA bacterial densities over $24 \mathrm{~h}$ time period.

The MRSA infection was treated with seven different exposures $(0.5-200$ folds of MIC $4 \mu \mathrm{g} / \mathrm{mL})$ of the FDC for $24 \mathrm{~h}$ to find PK/PD driver that can best describe the PD effect. It was noted that bacterial killing increase with increase in drug exposure till the saturation is achieved (Figure 3 and Table S1). After saturation (>50 fold MIC), there was no substantial increase in antibacterial effect. Thus 50 folds of MIC $(133.5 \mu \mathrm{g} / \mathrm{mL}$ of ceftriaxone and $66.75 \mu \mathrm{g} / \mathrm{mL}$ of vancomycin) was the lowest exposure for which maximum PD effect was observed; and thus the exposure (50x MIC) was selected for further dose optimization studies. 


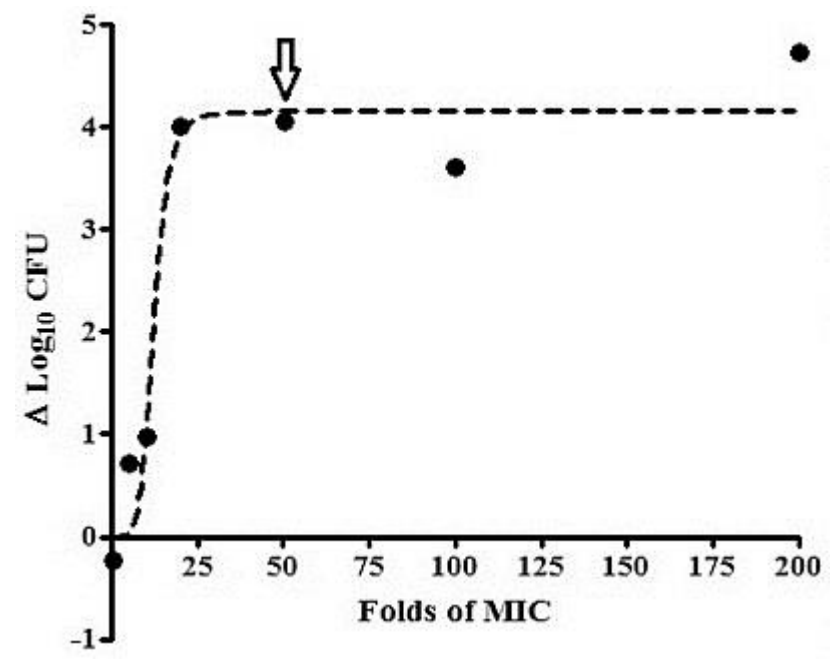

Figure 3. Total exposure in terms of fold in MICs was plotted against log fold change in bacterial density (CFU). Downward arrow represents the 50 fold MIC where the saturated pharmacodynamic response was observed. The same drug exposure was thus used in dose fractionation studies for dose optimization using in vitro studies.

Table 1. Pharmacokinetic parameters estimated in chemostat infection model and its comparison to reported values in literature

\begin{tabular}{|l|c|c|c|c|}
\hline \multirow{2}{*}{ Parameters } & \multicolumn{2}{|c|}{ As reported in literatures } & \multicolumn{2}{c|}{ Estimated from in vitro system } \\
\cline { 2 - 5 } & Ceftriaxone & Vancomycin & Ceftriaxone & Vancomycin \\
\hline Clearance (L/h) & $0.88 \pm 0.431[30]$ & $4.8 \pm 1.9[31]$ & $1.53 \pm 0.11$ & $3.59 \pm 0.11$ \\
\hline $\begin{array}{l}\text { Volume of } \\
\text { distribution (L) }\end{array}$ & $10.1 \pm 1.0[24]$ & $25.8[33]$ & $10.79 \pm 1.14$ & $22.3 \pm 0.67$ \\
\hline Half Life (h) & $5.9 \pm 0.7[24]$ & $4-6[34]$ & $4.83 \pm 0.71$ & $4.3 \pm 0.33$ \\
\hline
\end{tabular}

\section{Selection of pharmacokinetic/pharmacodynamic driver that can predict FDC's antibacterial efficacy}

The routine PK/PD indices i.e. AUC/MIC, $C_{\max } / \mathrm{MIC}$ and \%T>MIC were calculated for ceftriaxone and vancomycin individually, for all exposures. To understand of PK/PD driver responsible for therapeutic potential of the FDC, the FIC-time profiles were generated from concentration-time profile of both drugs for all doses as described above (See PK/PD analyses in Materials and Method section). The PK/PD indices i.e. $\mathrm{AUC}_{\text {comb }}, \mathrm{C}_{\text {max-comb }}, \% \mathrm{~T}>\mathrm{MIC}_{\text {comb }}$ and \%T $>\mathrm{FIC}$ were calculated from the FIC-time curves. All PK/PD indices and their respective logarithmic transformed values (excluding $\% \mathrm{~T}>\mathrm{MIC}_{\text {comb }}$ and $\% \mathrm{~T}>\mathrm{FIC}$ ) were plotted against the logarithmic reduction in bacterial densities of MRSA in 8, 9.5, 12 and $24 \mathrm{~h}$. The time periods of 8, 12 and $24 \mathrm{~h}$ were chosen considering thrice-a-day, twice-a-day and once-a-day regimen of the FDC. The time point of $9.5 \mathrm{~h}$ was included because second inoculum was added th this time point which might affect the net reduction in bacterial density.

Sigmoidal $E_{\max }$ type function (see materials and methods, equation 2) was used for fitting the exposureresponse dat; and coefficient of correlation $\left(R^{2}\right)$, and sigmoidal factor $(\gamma)$ were computed. Sigmoidal factor is the steepness of the sigmoidal curve and can suggest the predictability of the sigmodial model. For instance, when the steepness is high, the exposure-response relationship becomes steep enough for the all-or-none effect, which is not desired in identification of PK/PD drivers which is intended to correlate/simulate the dose-dependent sigmoidal drug effect. It must be noted that the intial 
concentration or $C_{\max }$ is predecided when the drug was injected in in vitro system, as shown by higher coefficients of correlation of $\log C_{\max }$ vs log $\Delta C F U s$ (Figure $4 \mathrm{~b}$ ). Thus, $C_{\max }$ curves were not further used to identify the PK/PD driver of the current FDC. Ceftriaxone individually $\left(R^{2}\right.$ of 0.82 for \%T>MIC) showed the time-dependent antibacterial effect of the drug, which also confirms with the literature [35] (Figure S1a). Since, AUC is directly dependent upon the drug concentration and time, AUC/MIC $\left(R^{2}=0.95\right)$ also showed good correlation with change in bacterial density (Figure S1a). For vancomycin, lower $R^{2}$ value of 0.38 for $\% \mathrm{~T}>\mathrm{MIC}$ vs log $\triangle \mathrm{CFUs}$ and higher $R^{2}$ value of 0.97 for log AUC/MIC vs log $\triangle$ CFUs suggest exposuredependent antibacterial effect and this is in agreement with the literature (Figure S1b) [33, 36-37].

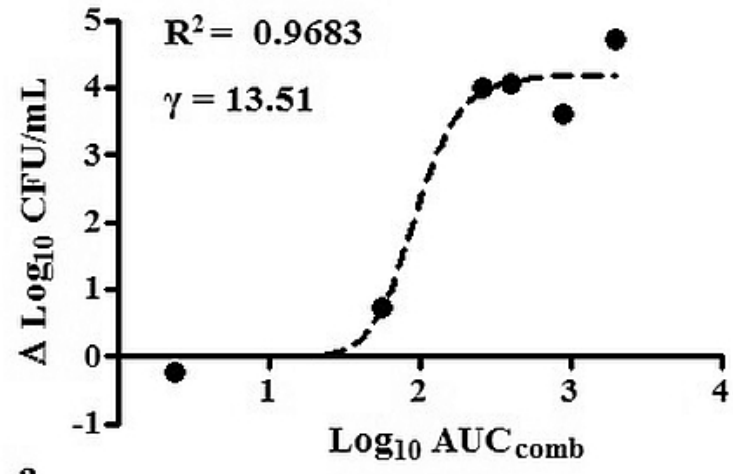

a.

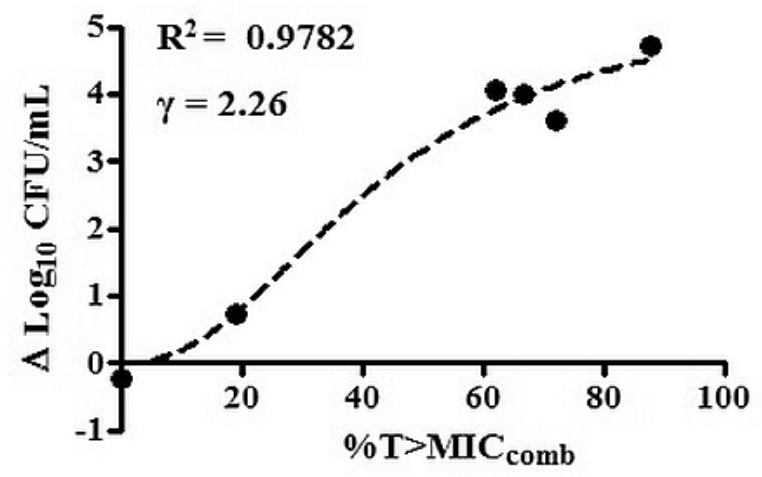

c.

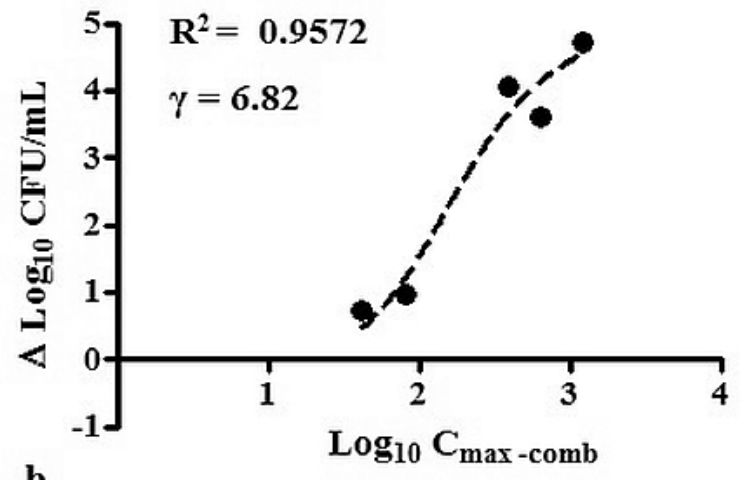

b.

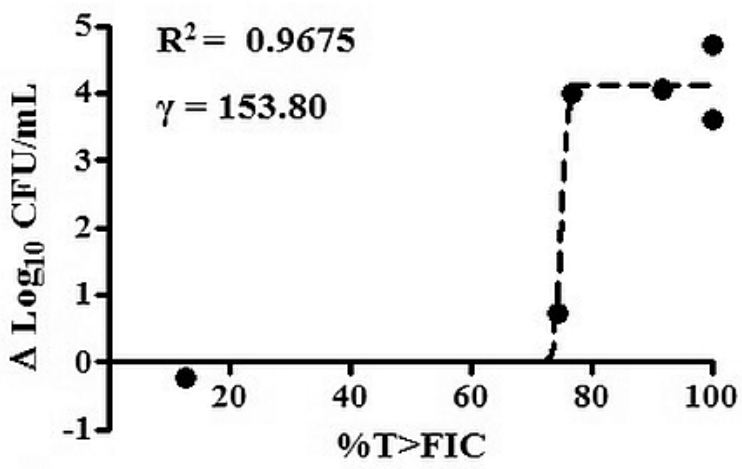

d.

Figure 4. The PK/PD indices i.e. $A U C_{\text {comb }}, C_{\text {max-comb }} \% \mathrm{~T}>\mathrm{MIC}_{\text {comb }}$ and $\% \mathrm{~T}>\mathrm{FIC}$ were calculated for FDC using $\mathrm{FIC}_{\text {comb }}$ curves. The log transformations were done for $\mathrm{AUC} / \mathrm{MIC}_{\text {comb }}$ and $C_{\text {max-comb }} / \mathrm{MIC}$. The log transformed $\mathrm{PK} / \mathrm{PD}$ indices and \%T>MIC were plotted against log fold change in bacterial density. The data was fitted with a sigmoidal $E_{\max }$ type function. Model fitted values of FDC's PK/PD index $A U C_{\text {comb }}(a), \log C_{\max \text {-comb }}(\mathrm{b})$, $\% \mathrm{~T}>\mathrm{MIC}_{\text {comb }}(\mathrm{c})$, and \% $>\mathrm{FIC}(\mathrm{d})$, and its relationship with log fold change in bacterial density were shown.

Since the PK/PD driver of ceftriaxone and vancomycin were different, FICs were used in order to combine the contribution of both drugs as per their concentration and MIC contribution in the FDC. The objective was to find the PK/PD index that can predict the antibacterial effect of the ceftriaxone/vancomycin (2/1) as a single unit. From FIC-t profiles, $\mathrm{AUC}_{\text {comb }}, \mathrm{C}_{\text {max-comb }}, \% \mathrm{~T}>\mathrm{MIC}_{\text {comb }}$, and $\% \mathrm{~T}>\mathrm{FIC}$ were calculated and plotted against bacterial killing (Figure 4). As shown in Figure 4c, the $R^{2}$ is highest for $\% \mathrm{~T}>\mathrm{MIC}_{\text {comb }}$ i.e. 0.98 with high predictability $(\gamma=2.262)$. The second best $\mathrm{R}^{2}$ of 0.97 was obtained from the plot of log $\mathrm{AUC}_{\text {comb }}$ vs $\log \Delta \mathrm{CFU}$ ( $\gamma=13.51$; good predictability) (Figure 4a). For \%T>FIC, $R^{2}$ was 0.97 but $\gamma$ was very high at 153.8 (Figure 4d). High value of sigmoidicity factor suggest all-or-none function of the system and thus decrease the predictability of model. Therefore, $\% \mathrm{~T}>\mathrm{MIC}$ comb was considered as a best PK/PD driver followed by $\mathrm{AUC}_{\text {comb }}$, to predict the PD effect of ceftriaxone/vancomycin $(2 / 1)$ in the study. 


\section{Probability target attainment levels}

After selection of PK/PD driver (i.e. \% $>\mathrm{MIC}_{\text {comb }}$ and $A \cup \mathrm{C}_{\text {comb }}$ ), the next objective was to identify a desired value of $\% \mathrm{~T}>\mathrm{MIC}_{\text {comb }}$ and $\mathrm{AUC}_{\text {comb }}$ that one should target to assure good therapeutic effect of the FDC. Thus, plot of \%T>MIC vs. $\Delta \log _{10} \mathrm{CFU}$ was evaluated to estimate desired \%T>MIC value (Figure $4 \mathrm{C}$ ). It was clearly observed that PD effect of the FDC become saturated after $60 \% \mathrm{~T}>\mathrm{MIC}$, prompting us to select 60 as a target value of PK/PD i.e. \%T>MIC comb for dose optimization. In other words, one can assure good therapeutic effect of the FDC, if the drug concentration in body remains above MIC for more than $60 \%$ of time of drug exposure. Using the same rationale and Figure 4a, the target value of 400 ( antilog of 2.6) was used for the second best PK/PD driver i.e. $\mathrm{AUC}_{\text {comb }}$.

\section{Dose optimization and recommendation}

The PK/PD drivers of FDC and its desired values were further utilized for dose optimization and recommendation using three approaches i.e. in vitro approach, PK/PD modeling and Monte Carlo simulations. These approaches use same piece of information and provide inferential data for dose optimization which vary from each other in terms of specificity of information. The first approach (in vitro system) mainly tells about the antibacterial potential of the FDC under a specific MIC. The PK/PD model is generally employed for dose individualization; whereas, Monte Carlo simulations broadly recommends dose regimen based on MIC of the target infection. All three approaches were utilized to understand the holistic view and are explained below in detail.

Dosage optimization using in vitro system: The PD effect being driven by the time-dependence of FDC, encouraged us to fractionate the dose, with an objective to increase the duration of exposure (above MIC) and decrease the overall concentration (to reduce side effects). For dose fractionation studies, dose exposure of $50 x$ MIC i.e. 133.5/66.75 $\mathrm{gg} / \mathrm{mL}$ (ceftriaxone/vancomycin) was selected. Considering the antibiotic half-life and MIC of drug against MRSA strains, the dose was fractionated to mimic a dosage regimen of once-a-day, twice-a-day, and thrice-a-day (Figure 5). Similar to dose escalation study, the in vitro system was used for these dosage regimens and results are shown in Figure 5 and Table S1. The antibacterial effect of the FDC was increased from once-a day dosing to twice-a-day dosage regimen in 24 hr time period ( 4.058 fold to 5.550 fold reduction in bacterial density). Further fractionation of dose in thrice-a-day dosing decreased the drug concentration below MIC for longer time period resulting in reduced 'effective' exposure time i.e. \%T>MIC and translated into sub-optimal 2.32 fold reduction in bacterial density. The selected dosage regimen roughly corresponds to $3 \mathrm{~g}$ FDC of ceftriaxone $(2 \mathrm{~g})$ and vancomycin $(1 \mathrm{~g})$, after correcting for protein binding of 80 and $40 \%$; and volume of distribution of $10.1 \mathrm{~L}$ and $25.8 \mathrm{~L}$ of both components i.e. ceftriaxone and vancomycin respectively was observed (Table 1 ). The MIC values remained same during $24 \mathrm{~h}$ drug-bacterial incubation period for all drug exposures (data not shown), thus ruling out the possibility of antimicrobial resistance development in MRSA isolates during in vitro studies. 


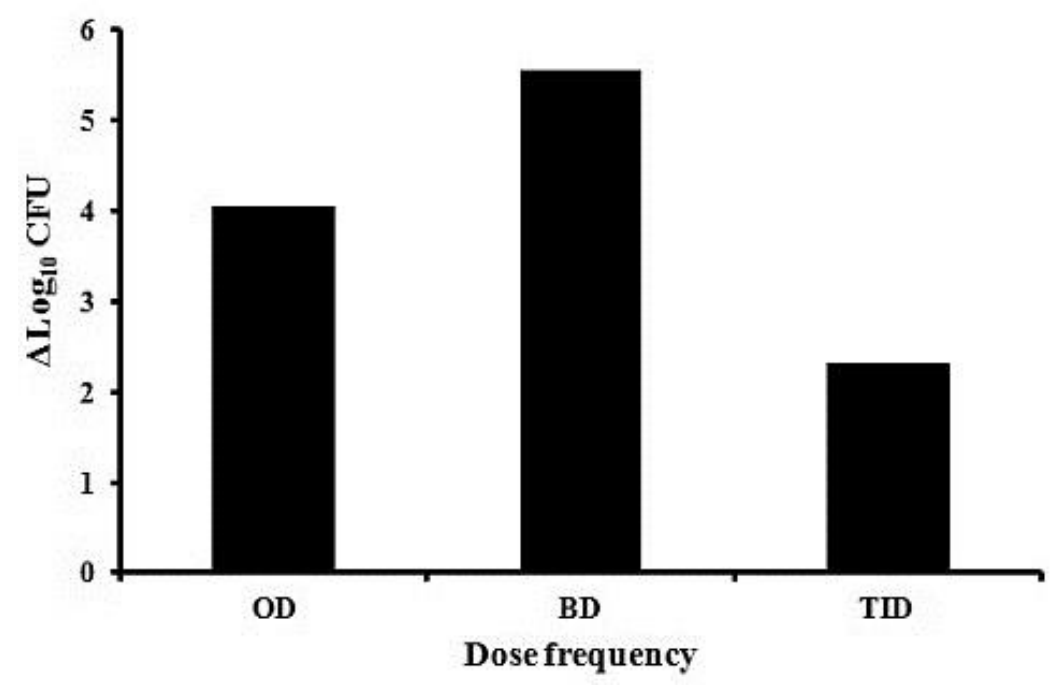

Figure 5. Selected 50 Fold MIC concentrations of the fixed dose combination of ceftriaxone/sulbactam (2/1) were fractioned into once-a-day (OD), twice-a-day (BD) and thrice-a-day (TID) dosage regimens, and were plotted against log fold change in bacterial density (CFU).

Dosage optimization using PK/PD modeling: PK/PD model development: The PK/PD model was developed using equation 3 of sub-section section 'antibacterial-PK/PD modeling' under 'Materials and Method' section. The net bacterial growth $\left(K_{\text {net }}\right)$ was determined from growth kinetics of bacteria without adding drug. For the determination of $E_{\max }, \mathrm{EC}_{50}$ and $\gamma$, dose response curve was constructed and analyzed using in vitro dilution system.

Depending upon the therapeutic action needed, the dose can be adjusted for enhanced PD effects using dose-response relationship (these relationships are reflections of the pharmacological actions of the drug). The MRSA infections were exposed to $25,38,50,75$ and $100 \%$ of dosage regimen in in vitro chemostat system and the corresponding logarithmic change in bacterial densities of $0.08,4,4.6,4.48$, and 5.55 were observed respectively. The dose response curve was plotted (Figure S2) and the data was fitted with sigmoidal function and $\mathrm{EC}_{50}$ of $37.46 \%$ was calculated (defined as a percentage of dose required to attain the 50 percent of $\mathrm{PD}$ effect). This $\mathrm{EC}_{50}$ value was used along with other parameters i.e. $E_{\max }, v$ to create the semi-mechanistic antibacterial bacterial-PK/PD model. The model might help in determining the antibacterial response at a given concentration (and in clinical settings at given dose) and could be extrapolated to estimate dose requirements for an identified patient's bacterial load in clinical settings.

PK/PD model validation: The final PK/PD equation was validated to check its suitability in dose recommendation using SCIENTIST. The experimental data for dose escalation studies were fitted using equation-3. The goodness of fit and $95 \%$ confidence interval of simulated data were evaluated for first $8 \mathrm{~h}$ (before the addition of second inoculum). Simulations after $8 \mathrm{~h}$ of drug exposure were not shown in case of dose-escalation study in order to focus mainly on initial exposure of drug, where most of the bacterial killing occurred. The semi-mechanistic-PK/PD model predictions were improved with an increase in dose exposure. For instance, $R^{2}$ values for the fitted values of dose exposures of 20x, 50x, 100x and 200x MIC were $0.633,0.856,0.964$ and 0.965 respectively (Figure $6 a, 6 b, 6 c$ and $6 \mathrm{~d}$ ) suggesting better predictability of model at comparitively higher exposures. Additionally, most of the experimental data points were under $95 \%$ confidence interval of simulated data, which further supports the validity of PK/PD model. 

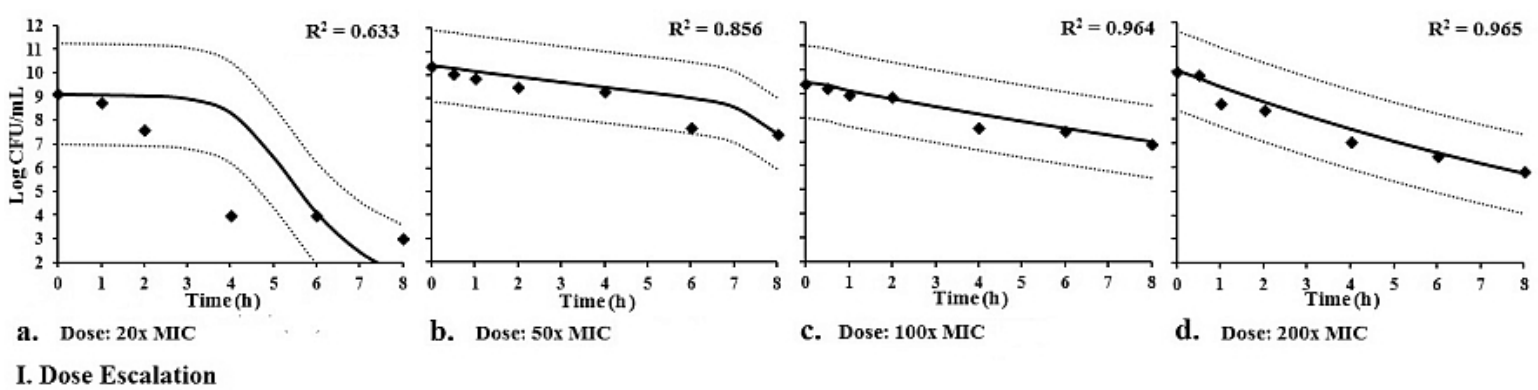

b. Dose: 50x MIC

c. Dose: $100 \mathrm{x} \mathrm{MIC}$

d. Dose: $200 \times$ MIC

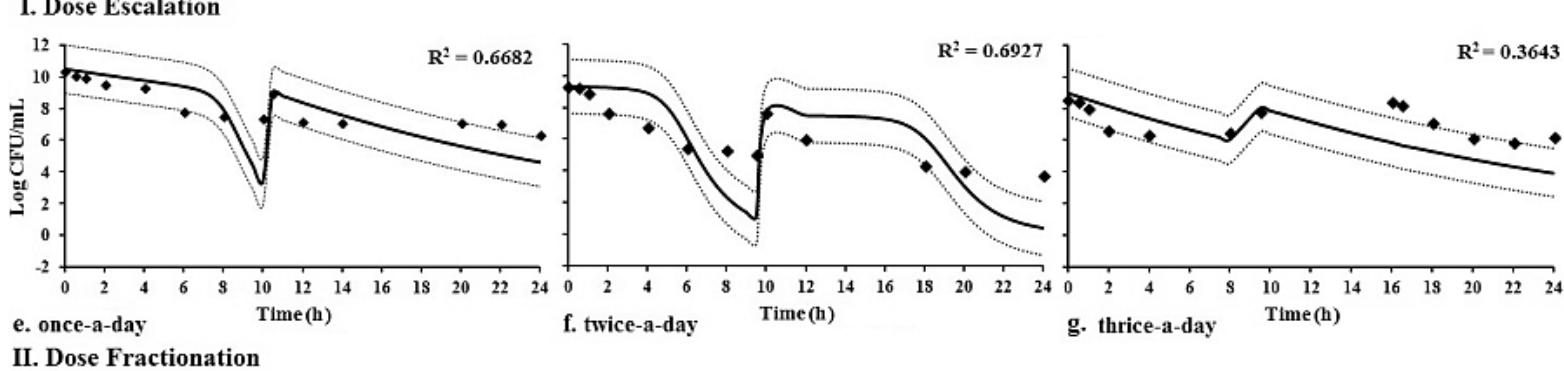

II. Dose Fractionation

Figure 6. The experimental data was fitted with the antibacterial-PK/PD model using non-linear least-square regression program SCIENTIST. (I) Validation based on dose escalation studies data (a, b, c, d):The data of dose escalation from 20 folds to 200 folds of MIC was validated with the model; (II) Validation based on dose fractionation studies data: The data of dose fractionation i.e. once-a-day (e), twice-a-day(f) and thrice-a-day (g) was validated with the model.

The model (equation 3) was further applied to experimental data of dose fractionation. Since, dose frequency is a main factor in dose fractionation data, model predictions were extended to 24 hours. The observed values of experiments and predicted values of model were plotted for all three dosage regimens i.e. once-a-day, twice-a-day and thrice-a-day (Figure $6 \mathrm{e}, 6 \mathrm{f}$ and $6 \mathrm{~g}$ ). The addition of second inocula at $9.5 \mathrm{~h}$ had raised the CFU counts, as shown by corresponding downward peak in all three cases. Also as mentioned before, model was weak for lower concentrations of drug, as observed in 20-24 h of once-a-day dosing (Figure 6e) and 16-24 h of thrice-a-day dosing (Figure 6g) of ceftriaxone/vancomycin (2/1). Therefore, weak correlation was observed in all three cases as compared to dose escalation studies. The correlation coefficents increases from 0.6682 of once-a-day dosing to 0.6927 of twice-a-dosing of the FDC of antibiotics. Further increase in dose fractionation reduces drastically the correlation coefficient to 0.3643 , which suggests the model weakness in predicton of lower drug concentrations. However, the model was appropiate for the twice-a-day dose frequency, which was also the best dosage regimen for ceftriaxone/vancomycin (2/1) FDC combination. The same conclusion was derived from the $95 \%$ confidence interval of simulated data. Most of the data of twice-a-day dosing were in the upper and lower limits of confidence interval, followed by once-a-day dosing (Figure 6). Again in case of thrice-a-day dose frequency, predicted values were poorly correlated with the experimental values (Figure 6g).

Dose optimization using Monte Carlo simulations: The PK-PD studies were done using the fixed values of primary PK parameters and MIC; which did not account the PK variability of a clinical population. In order to account for the randomness/variability in human PKs and MICs, Monte Carlo simulations of 1000 subjects were done to determine how likely the given exposure of FDC would achieve the target $\% \mathrm{~T}>\mathrm{MIC}_{\text {comb }}$ or $\mathrm{AUC}_{\text {comb }}$ (reported as PTA). The PK parameters utilized were clearance of $0.88+/-0.431 \mathrm{~L} / \mathrm{h}$ and $4.8+/-1.9 \mathrm{~L} / \mathrm{h}$ and elimination rate constant of 0.113 and $0.11 \mathrm{~h}^{-1}$ for ceftriaxone and vancomycin respectively, on the basis of literature [30,31]. Monte Carlo simulations were done for both PK/PD indices i.e. $\mathrm{AUC}_{\text {comb }}$ and \%T>MIC comb (Figure $7 \mathrm{a}$ and $7 \mathrm{~b}$ ). The selection of the PTA target set-points (as described previously) were done based on the $\mathrm{AUC}_{\text {comb }}$ and $\% \mathrm{~T}>\mathrm{MIC}_{\text {comb }}$ values which correlates best with maximal bacterial killing effect ( 4 fold log reduction in bacterial density). In the present study, the PTA for AUC $_{\text {comb }}$ 
was defined as percentage of the simulated subjects in which $A \cup C_{\text {comb }}$ was more than 400 [2]. For $\% \mathrm{~T}>\mathrm{MIC}_{\text {comb }}$, the PTA was defined as percentage of simulated subjects showing $\% \mathrm{~T}>\mathrm{MIC}_{\text {comb }}$ of more than 60.

Focusing on PTA of $A \cup C_{\text {comb }} \geq 400$, ceftriaxone/vancomycin (2/1) had achieved almost $98 \%$ PTA for all doses against the strains for which it exhibits MIC of $1 \mu \mathrm{g} / \mathrm{mL}$ (Figure 7a). Also for the MIC of $4 \mu \mathrm{g} / \mathrm{mL}$, the lowest dose that can achieve the PTA of more than $90 \%$ was $1.5 \mathrm{~g}$ twice dosing. These strains were categorized as susceptible to the FDC. Further increase in MIC i.e. $8 \mu \mathrm{g} / \mathrm{mL}$ lowered the PTA ( $31 \%$ at $1.5 \mathrm{~g}$ twice-a-day) and could be improved with higher dose of the FDC ( $\approx 90 \%$ PTA at 3.0g twice-a-day). However, strains corresponding to MIC of 16,32 and $64 \mu \mathrm{g} / \mathrm{mL}$ required large amount of FDC dose $(>9 \mathrm{~g})$ to attain moderate PTA (Figure 7a). These strains were resistant to the therapeutic dose of FDC.

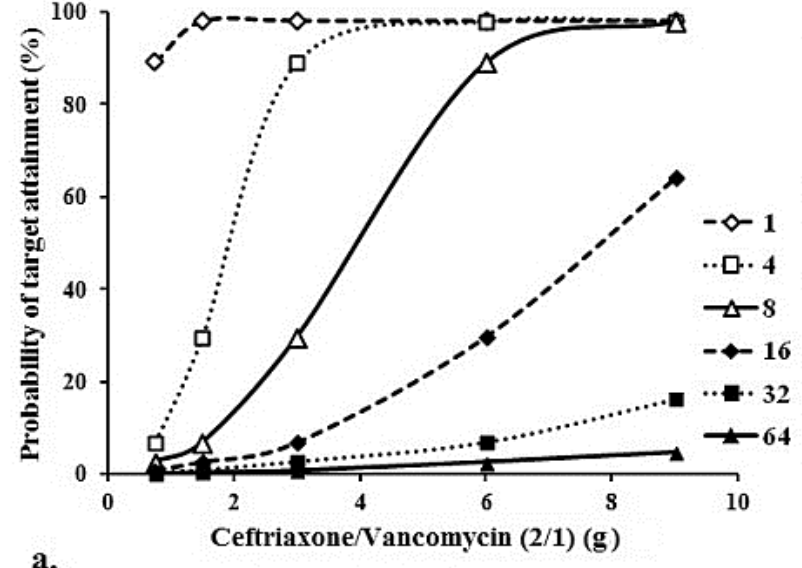

a.

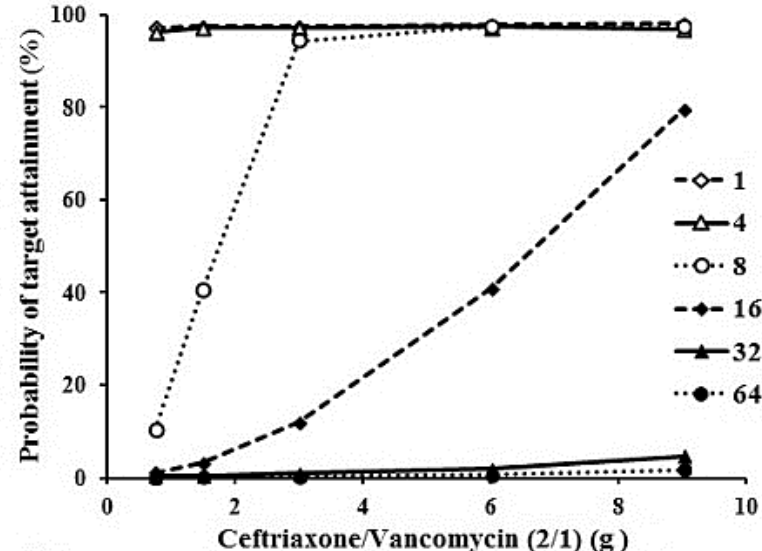

b.

Figure 7. Clinical breakpoint estimation $(a, b)$ : Monte Carlo simulations for 1000 subjects and PTA of both $\mathrm{PK} / \mathrm{PD}$ indices i.e. $\mathrm{AUC}_{\text {comb }}(\mathrm{a})$ and $\% \mathrm{~T}>\mathrm{MIC}_{\text {comb }}(\mathrm{b})$ was evaluated in the simulated subjects. The PTA for $A \cup C_{\text {comb }}$ was defined as percentage of the simulated patients in which $\mathrm{AUC}_{\text {comb }}$ was more than 400 . Similarly

PTA for $\% \% \mathrm{~T}>\mathrm{MIC}_{\text {comb }}$ was 60 . It was determined that how likely the FDC dose of $0.75,1.5,3,6$, and $9 \mathrm{gm}$ would achieve $\mathrm{AUC}_{\text {comb }} / \mathrm{MIC}_{\text {comb }}>400$ and $60 \% \mathrm{~T}>\mathrm{MIC}$ comb at different values of MIC i.e. 1, 4, 8, 16, 3, $64 \mu \mathrm{g} / \mathrm{mL}$.

The same results were mirrored with more clarity in $\% \mathrm{~T}>\mathrm{MIC}_{\text {comb }}$ based Monte Carlo simulations (Figure 7b). It must be mentioned that the PK/PD index i.e. \%T>MIC comb was best PK/PD driver for FDC. As shown in Figure $7 b$, the combination was very effective with PTA of $\approx 99 \%$ at all dose strengths, for the strains corresponding to MIC of 1 and $4 \mu \mathrm{g} / \mathrm{mL}$. For MIC of 8 and $16 \mu \mathrm{g} / \mathrm{mL}$, higher dose of FDC was required to revive its efficacy against the bacterial infection. Interestingly, the PD effect against the strains of MIC of 8 $\mu \mathrm{g} / \mathrm{mL}$, was tilted more toward susceptible range of MIC (1 and $4 \mu \mathrm{g} / \mathrm{mL})$, whereas strains corresponding to MIC of $16 \mu \mathrm{g} / \mathrm{mL}$ was closer to resistant ones. Following the same trend observed in case of $\mathrm{AUC}_{\text {comb }}$ (Figure 7a), the FDC was ineffective at all drug exposures for the MIC of 32 and $64 \mu \mathrm{g} / \mathrm{mL}$. For these resistant strains, standard dose of $1.5 \mathrm{~g}$ and $3.0 \mathrm{~g}$ twice-a-dose were sub-optimal and higher doses were needed to achieve the antibacterial effect. Thus, the strains corresponding to the MIC of 1-4 $\mu \mathrm{g} / \mathrm{mL}, 8-16$ $\mu \mathrm{g} / \mathrm{mL}$ and $32-64 \mu \mathrm{g} / \mathrm{mL}$ were susceptible, intermediate and resistant respectively to the FDC of ceftriaxone/vancomycin (2/1). This categorization is also defined as clinical breakpoints, which separates resistant bacterial strains from the sensitive ones against a drug in terms of its MIC values. The clinical breakpoints obtained from Monte Carlo simulation, were compared with MIC breakpoints obtained from in-house study on 500 clinical isolates of MRSA (MIC and AST breakpoint estimation; Table-S2). The results were in agreement with each other, suggesting promising outcomes in future clinical studies of the FDC ceftriaxone/vancomycin (2/1).

These clinical breakpoints give a significant advantage over infections wherein vancomycin alone is 
showing therapeutic limitations. For example the susceptibility of MRSA for FDC (vancomycin + ceftriaxone) is 2 fold higher than vancomycin (MIC $\leq 4 \mu \mathrm{g} / \mathrm{mL}$ for FDC and $\leq 2 \mu \mathrm{g} / \mathrm{mL}$ for vancomycin) alone. In addition to MRSA susceptibility, the FDC provides broader antibacterial coverage (active against both gram positive and negative bacteria). The better clinical reach will help in tackling vancomycin mediated resistance more effectively. The cost-effectiveness study of the FDC with respect to other commercially available antibiotics is currently under investigation, as previously done by Sharma et al. on similar FDCs [38].

Our study mainly focused on in vitro PK/PD relationships of FDC antibiotics, however generalizing the inferences of this study to all FDC antibiotics will not be prudent. Also, the in vitro-in vivo translation, and finally its applicability in clinical settings still needs to be evaluated. We have focused on only one bacterial isolate, and we understand that when more clinical isolates with different resistance patterns will be studied, the exposure targets may get modified. However, the results of this study not only give the starting point to use the FDC clinically but will also refine the clinical study and design for further clinical research work.

\section{Conclusions}

To summarize, in vitro system was used to estimate PK parameters and PD effects for different exposure of the FDC of ceftriaxone/vancomycin (2/1). The best antibacterial effect was obtained from the exposure of $50 \mathrm{x} \mathrm{MIC}_{\text {comb }}$ which when fractioned to twice-daily dosing showed maximum reduction in bacterial densities. The semi-mechanistic PK/PD model incorporating bacterial kinetics and drug's PK/PD relationship showed good predictability (very week predictability at lower exposures) at therapeutic exposures. Taking into the consideration the randomness and PK variability of subjects, Monte Carlo simulations revealed that clinical breakpoints of FDC for susceptible, intermediate and resistant strains were $\leq 4,8-16$, and $\geq 32 \mu \mathrm{g} / \mathrm{mL}$, respectively. These breakpoints were 2 fold higher than vancomycin alone treatments. The same breakpoint values in in vitro (MIC and AST breakpoint estimation) and simulated clinical population suggests that FDC combination exhibits same PD effect in vitro that it should show in clinical population. Thus, the study corroborates the validity of antibacterial effect of FDC ceftriaxone/vancomycin $(2 / 1 \mathrm{w} / \mathrm{w} /)$ in simulated population with unique advantage of giving both components together in similar dosing frequencies.

Acknowledgements: This research was funded by Venus Remedies Ltd. Special thanks are extended to Analytical and Microbiological department of Venus Remedies for analyzing the samples.

\section{References}

[1] E. Klein, D.L. Smith, R. Laxminarayan, Emerging Infectious Disease 13(12) (2007) 1840-1846.

[2] F.C. Tenover, R.C. Moellering, Clinical Infectious Diseases 44(9) (2007) 1208-1215.

[3] B.P. Howden, P.D. Johnson, P.B. Ward, T.P. Stinear, J.K. Davies, Antimicrobial Agents and Chemotherapy 50(9) (2006) 3039-3047.

[4] P.G. Charles, P.B. Ward, P.D. Johnson, B.P. Howden, M.L. Grayson, Clinical Infectious Diseases 38(3) (2004) 448-451.

[5] E.M. O'Shaughnessy, J. Meletiadis, T. Stergiopoulou, J.P. Demchok, T.J. Walsh, Journal of Antimicrobial Chemotherapy 58(6) (2006) 1168-1176.

[6] E.I. Nielsen, L.E. Friberg, Pharmacological Reviews 65(3) (2013) 1053-1090.

[7] E.I. Nielsen, A. Viberg, E. Lowdin, O. Cars, M.O. Karlsson, M. Sandstrom, Antimicrobial Agents and Chemotherapy 51(1) (2007)128-136. 
[8] T. Katsube, Y. Yano, Y. Yamano, T. Munekage, N. Kuroda, M. Takano, Journal of Pharmaceutical Sciences 97(9) (2008) 4108-4117.

[9] E.I. Nielsen, O. Cars, L.E. Friberg, Antimicrobial Agents and Chemotherapy 55(10) (2011) 4619-4630.

[10] B.D. VanScoy, R.E. Mendes, M. Castanheira, J. McCauley, S.M. Bhavnani, R.N. Jones, L.V. Friedrich, J.N. Steenbergen, P.G. Ambrose, Antimicrobial Agents and Chemotherapy 58(10) (2014) 6024-6031.

[11] V.D. Sharma, A. Singla, M. Chaudhary, M. Taneja, International Journal of Pharmaceutical Sciences and Research 7(4) (2016) 1503-1512.

[12] S.A. Van Wart, D.R. Andes, P.G. Ambrose, S.M. Bhavnani, Diagnostic Microbiology and Infectious Disease 63(4) (2009) 409-414.

[13] A. Ogden, M. Kuhn, M. Dority, S. Buist, S. Mehrens, T. Zhu, D. Xiao, J.R. Miller, D. Hanna, Antimicrobial Agents and Chemotherapy 56(1) (2012) 124-129.

[14] K.D. Leuthner, C. Vidaillac, C.M. Cheung, M.J. Rybak, Antimicrobial Agents and Chemotherapy 54(9) (2010) 3799-3803.

[15] V.D. Sharma, A. Singla, M. Chaudhary, M. Taneja, International Journal of Pharmacy and Pharmaceutical Sciences 8(7) (2016) 346-353.

[16] J.G. den Hollander, J.W. Mouton, H.A. Verbrugh, Antimicrobial Agents and Chemotherapy 42(4) (1998) 744-748.

[17] V.D. Sharma, A. Singla, M. Chaudhary, M. Taneja, AAPS PharmSciTech 17(5) (2016) 1192-1203.

[18] CLSI. Performance standards for antimicrobial disk susceptibility tests. CLSI M02-A12 (2015)

[19] A.S. Tariq, J. Kumar, D. Reddy, M. Chaudhary, R.K. Singh, Science Asia (36) (2010) 297-304.

[20] B.J. McGrath, K.C. Lamp, M.J. Rybak, Antimicrobial Agents and Chemotherapy 37 (9) (1993) 19311937.

[21] J. Gloede, C. Scheerans, H. Derendorf, C. Kloft, Journal of Antimicrobial Chemotherapy 65(2) (2010) 186-201.

[22] A.J. Larsson, K.J. Walker, J.K. Raddatz, J.C. Rotschafer, Journal of Antimicrobial Chemotherapy 38(4) (1996) 589-597.

[23] J.H. Martin, R. Norris, M. Barras, J. Roberts, R. Morris, M. Doogue, G.R. Jones, Clinical Biochemist Reviews 31(1) (2010) 21-24

[24] I.H. Patel, S. Chen, M. Parsonnet, M.R. Hackman, M.A. Brooks, J. Konikoff, S.A. Kaplan, Antimicrobial Agents and Chemotherapy 20(5) (1981) 634-641.

[25] W.J. Jusko, Journal of Pharmaceutical Sciences 60(6) (1971) 892-895.

[26] J.W. Mouton, A.A. Vinks, N.C. Punt, Antimicrobial Agents and Chemotherapy 41(4) (1997) 733-738.

[27] Y. Harigaya, J.B. Bulitta, A. Forrest, G. Sakoulas, A.J. Lesse, J.M. Mylotte, B.T. Tsuji, Antimicrobial Agents and Chemotherapy 53(9) (2009) 3894-3901.

[28] A. Nolting, T. Dalla Costa, K.H. Rand, H. Derendorf, Pharmaceutical Research 13(1) (1996) 91-96.

[29] J.B. Bulitta, N.S. Ly, J.C. Yang, A. Forrest, W.J. Jusko, B.T. Tsuji, Antimicrobial Agents and Chemotherapy 53(1) (2009) 46-56.

[30] D. Garot, R. Respaud, P. Lanotte, N. Simon, E. Mercier, S. Ehrmann, D. Perrotin, P.F. Dequin, C. Le Guellec, British Journal of Clinical Pharmacology 72(5) (2011) 758-767.

[31] J.Y. Chung, S.J. Jin, J.H. Yoon, Y.G. Song, Journal of Korean Medical Sciences 28(1) (2013) 48-54.

[32] V.H. Tam, M. Nikolaou, PLoS Computational Biology 7(1) (2011) e1001043.

[33] M.J. Rybak, Clinical Infectious Diseases 42 (2006) Suppl 1:S35-39.

[34] R.C. Baselt, Disposition of toxic drugs and chemicals in man. 9 edn. (2011) Biomedical Publications, Foster City, CA

[35] J.D. Turnidge, Clinical Infectious Diseases 27(1) (1998) 10-22

[36] S.J. Vandecasteele, A.S. De Vriese, E. Tacconelli, Journal of Antimicrobial Chemotherapy 68(4) (2013) 743-748. 
[37] G.R. Matzke, G.G. Zhanel, D.R. Guay, Clinical Pharmacokinetics 11(4) (1986) 257-282.

[38] V.D. Sharma, M. Chaudhary, M. Taneja, European Journal of Pharmaceutical and Medical Research 3(6) (2016) 454-462.

(C2016 by the authors; licensee IAPC, Zagreb, Croatia. This article is an open-access article distributed under the terms and conditions of the Creative Commons Attribution license (http://creativecommons.org/licenses/by/3.0/) (cc) B BY 


\section{Supplemental materials}

Supplement Table 1: Bacterial density reduction at various antibiotic exposures

\begin{tabular}{|l|c|c|}
\hline Concentration used (Ceftriaxone/Vancomycin $\mu \mathrm{g} / \mathrm{mL}$ ) & Folds of MIC & Log $\Delta$ CFU \\
\hline $1.335 / 0.667$ & 0.5 & -0.234 \\
\hline $13.35 / 6.675$ & 5 & 0.716 \\
\hline $26.7 / 13.35$ & 10 & 0.977 \\
\hline $53.4 / 26.7$ & 20 & 4.000 \\
\hline $133.5 / 66.75$ & 50 & 4.058 \\
\hline $267.0 / 133.5$ & 100 & 3.609 \\
\hline $534.0 / 267.0$ & 200 & 4.719 \\
\hline $66.75 / 33.375$ BD (total exposure: $133.5 / 66.75)$ & 50 & 5.55 \\
\hline $44.5 / 22.25$ TID (total exposure: $133.5 / 66.75)$ & 50 & 2.32 \\
\hline
\end{tabular}

The concentrations of individual component of FDC and bacterial CFU-time profiles were obtained from in vitro chemostat model. Bacterial killing increase with increase in drug exposure till the saturation is achieved i.e. from 0.5 -50 folds of MIC and after saturation, further increase in drug concentration from 50 to 200 folds of MIC, there was no substantial increase in anti-bacterial effect of antibiotic combination. Further, selected total exposure $(133.5 / 66.75 \mu \mathrm{g} / \mathrm{mL})$ of 50 fold MIC was fractionated into twice daily and thrice daily regimens. The twice daily regimen showed enhanced bacterial killing.

CFU - colony forming units; FDC - fixed dose combination; MIC - minimum inhibitory concentration

Supplement Table 2: Clinical, MIC and AST breakpoints of 150 bacterial isolates of MRSA

\begin{tabular}{|l|c|c|c|}
\hline Breakpoints & Susceptible & Intermediate & Resistant \\
\hline Clinical $(\mu \mathrm{g} / \mathrm{mL})$ & $\leq 4$ & $8-16$ & $\geq 32$ \\
\hline $\mathrm{MIC}(\mu \mathrm{g} / \mathrm{mL})$ & $\leq 4$ & $8-16$ & $\geq 32$ \\
\hline AST $(\mathrm{mm})$ & $<14$ & $14-17$ & $>17$ \\
\hline
\end{tabular}

The in vitro antibacterial activity of FDC was evaluated against MRSA. The clinical breakpoint was estimated from Monte Carlo simulations. The MIC breakpoints were determined based on target attainment of PK/PD index \%T>MIC ( $\approx 50 \%$ T $>$ MIC). The AST breakpoints were determined using least square regression analysis of zone diameter values with the corresponding MIC values of the FDC combination against 150 bacterial isolates of MRSA.

AST - antimicrobial susceptibility test; CFU - colony forming units; FDC - fixed dose combination; MIC minimum inhibitory concentration; MRSA - methicillin resistant Staphylococcus aureus; PK/PD pharmacokinetic/pharmacodynamic; \% T>MIC - \% time for which the levels of antibiotic in serum/plasma exceed the MIC. 

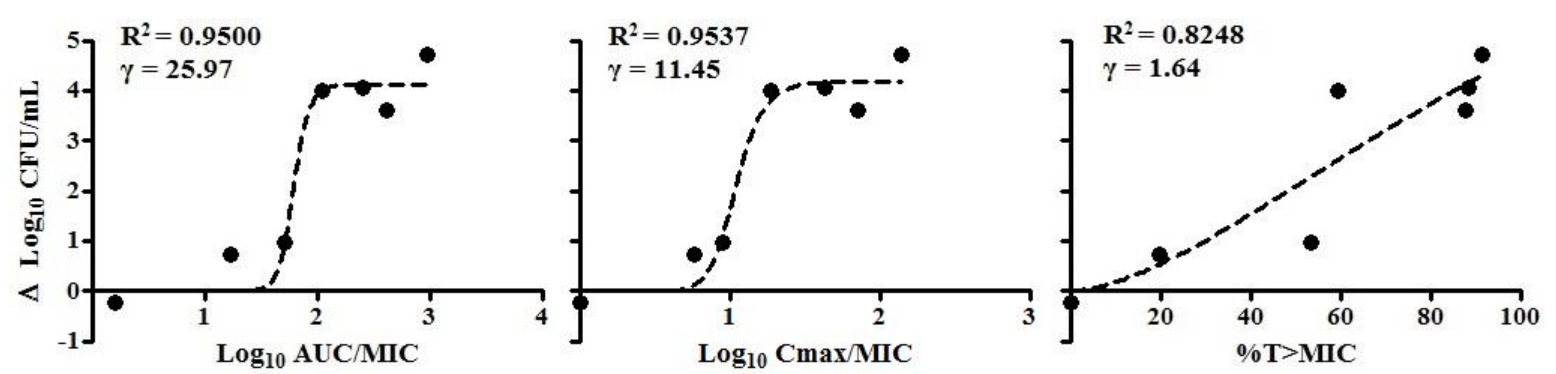

a. Ceftriaxone
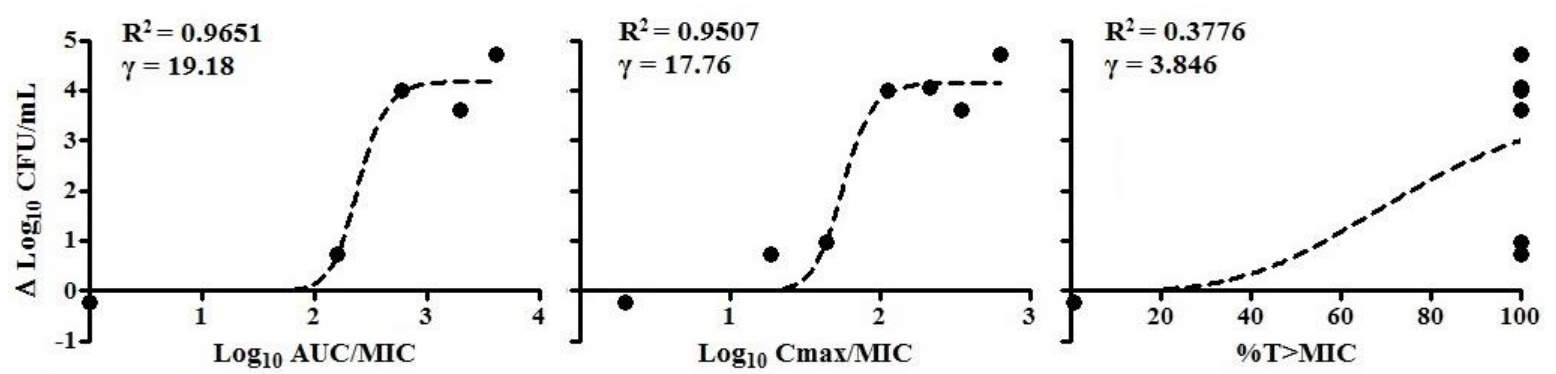

b. Vancomycin

Supplement Figure 1: Pharmacokinetic/pharmacodynamic indices of individual components of FDC and their relationship with log fold change in bacterial density

The PK/PD indices i.e. AUC/MIC, $C_{\max } / \mathrm{MIC}$ and \%T>MIC were calculated for both ceftriaxone and vancomycin using their respective PK parameters. The log transformations were done for AUC/MIC and $C_{\max } / \mathrm{MIC}$. The log transformed PK/PD indices and \%T>MIC were plotted against log fold change in bacterial density. The data was fitted with a sigmoidal $E_{\max }$ type function $E=E_{0}-\frac{P D_{\max } X^{\text {nulu }}}{\boldsymbol{X}^{\text {hill }}+E X_{50}^{\text {hill }}}$.

The selection of PK/PD driver was based on coefficient of correlation $\left(R^{2}\right)$, and sigmoidal factor ( $\gamma$ : Hill factor) values. A. Model fitted values of ceftriaxone PK/PD indices and its relationship with log fold change in bacterial density; B. Model fitted values of vancomycin PK/PD indices and its relationship with log fold change in bacterial density

$E-P D$ endpoint i.e. bacterial density calculated as change in $\log _{10} C F U / m L ; E_{0}$ - the baseline effect i.e. PD endpoint without antibiotic treatment; $P D_{\max }$ - maximum effect; $X-P K / P D$ index; $E X_{50}$ - magnitude of $X$ that is needed to achieve $50 \%$ of the $P D_{\max }$ Hill - sigmoidicity factor; FDC - fixed dose combination; $P D$ pharmacodynamic; PK - pharmacokinetic; MIC - minimum inhibitory concentration, \% T>MIC - \% time for which the levels of antibiotic in serum/plasma exceed the MIC; AUC/MIC - area under the concentrationtime curve/MIC ratio; $C_{\max } / \mathrm{MIC}$ - peak plasma concentration/MIC ratio. 


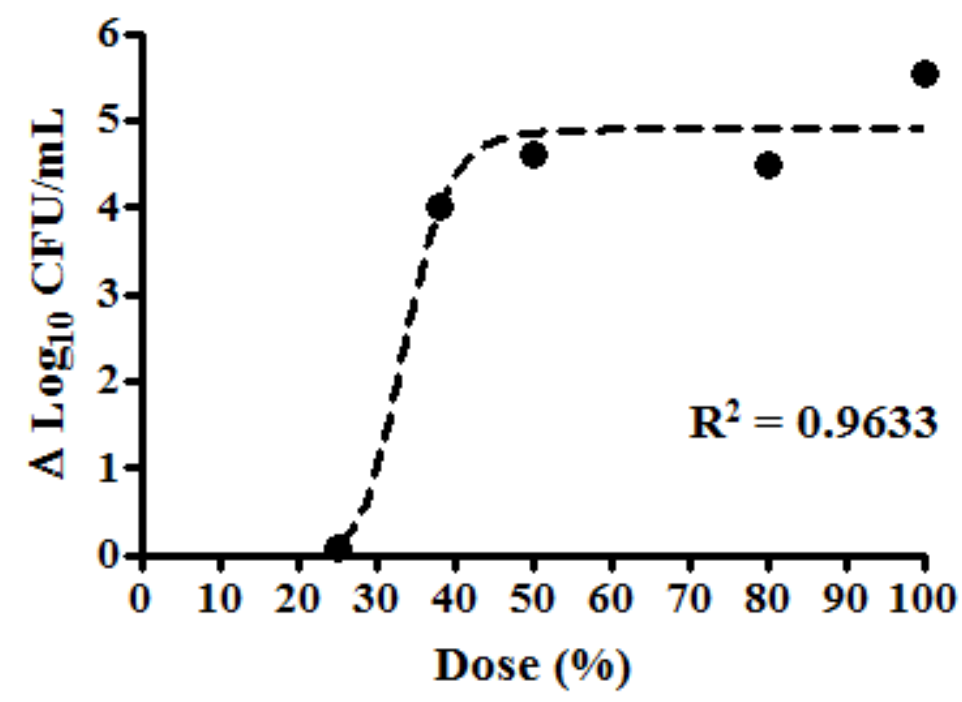

Supplement Figure 2: Dose response curve of optimized twice daily regimen of FDC

The best exposure from dose escalation study fractionated twice daily dosage regimen of FDC enhanced bacterial killing. The twice daily regimen at $75,50,38$, and $25 \%$ of original concentration and the change in bacterial density in $24 \mathrm{~h}$ was plotted to prepare the DRC. The DRC was plotted between the dose percentage and log reduction in bacterial density and sigmoidal function was used for curve fitting. The model showed good correlation $\left(R^{2}=0.9633\right)$ and $E C_{50}$ of $37.46 \%$ was estimated.

$E C_{50}$ - percentage of dose required to attain the 50\% of PD effect; FDC - fixed dose combination; $D R C$ - dose response curve; PD pharmacodynamics.

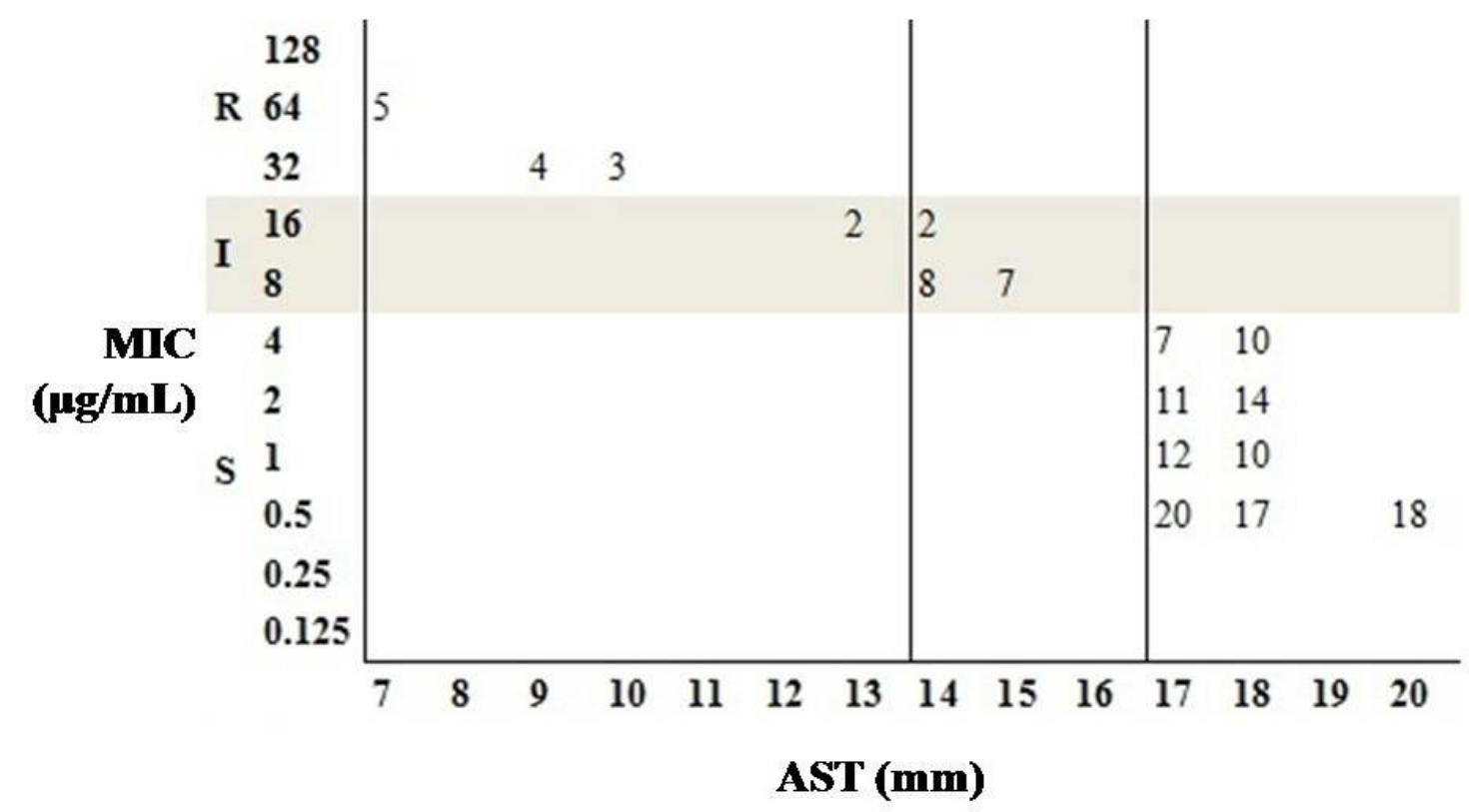

Supplement Figure 3: Breakpoint analysis of fixed dose combination of ceftriaxone and vancomycin against 150 MRSA strains

The in vitro susceptibility tests categorize the bacterial isolates into three categories: susceptible (S), intermediate (I), and resistant (R). The MIC breakpoints was determined on the basis of attainment of $\mathrm{PK} / \mathrm{PD}$ index $\% \mathrm{~T}>\mathrm{MIC} \approx 50 \% \mathrm{~T}>\mathrm{MIC}$. The plot of MIC values of various strains against the zone diameter (AST) for MRSA and segregated based on the MIC breakpoints of $4 \mu \mathrm{g} / \mathrm{mL}$ (S) and $32 \mu \mathrm{g} / \mathrm{mL}$ (R). 
AST - antimicrobial susceptibility; MIC - minimum inhibitory concentration, \% T>MIC - \% time for which the levels of antibiotic in serum/plasma exceed the MIC

(C2016 by the authors; licensee IAPC, Zagreb, Croatia. This article is an open-access article distributed under the terms and conditions of the Creative Commons Attribution license (http://creativecommons.org/licenses/by/3.0/) (cc) BY 